Monatsschr Kinderheilkd 2012 • 160:1250

DOI 10.1007/s00112-012-2839-6

(c) Springer-Verlag Berlin Heidelberg 2012

\section{K. Tenbrock}

Abteilung für Pädiatrische Rheumatologie, Klinik für Kinder und Jugendmedizin, Klinikum, RWTH Aachen

\title{
Erratum zu: Therapie der juvenilen idiopathischen Arthritis (JIA) - Neue Therapiekonzepte: Biologicals
}

\section{Monatsschr Kinderheilkd (2012) 160:224-230 http://dx.doi.org/10.1007/s00112-011-2546-8}

Leider ist es in diesem Beitrag zu fehlerhaften Angaben bezüglich Dosierung und Zulassung des Medikaments Adalimumab gekommen. In . Tab. 1 sowie im Kapitel „Adalimumab/Dosierung“ wurde die Dosierung von Adalimumab zur Behandlung von polyartikulärer JIA, enthesitisassoziierter JIA, oligoartikulärer JIA und Uveitis bei Patienten $<30 \mathrm{~kg}$ mit $24 \mathrm{mg} / \mathrm{m}^{2} \mathrm{KOF}$ (Körperoberfläche), bei Patienten $>30 \mathrm{~kg}$ mit $20 \mathrm{mg} / \mathrm{m}^{2}$ KOF mit maximal $40 \mathrm{mg}$ alle 2 Wochen s.c. mit einer Zulassung ab dem 4. Lebensjahr angegeben.

Diese Dosierungsangaben entsprechen nicht den Angaben der Fachinformation. Diese wurde ebenso wie die $\mathrm{Zu}$ lassung korrigiert, wie in $\bullet$ Tab. 1 dargestellt. Korrekt müssten die Angaben folgendermaßen lauten: Bei Patienten im Alter von 4 bis 12 Jahren $24 \mathrm{mg} / \mathrm{m}^{2} \mathrm{KOF}$, aber maximal $40 \mathrm{mg}$; bei Patienten im Alter von 12 bis 17 Jahren $40 \mathrm{mg}$ alle 2 Wochen s.c. Das Medikament ist zur Therapie von polyartikulärer JIA ab dem 4 . Lebensjahr zugelassen.

Wir bitten diesen Fehler zu entschuldigen und künftig die korrigierten Angaben zu beachten.

\section{Korrespondenzadresse}

\section{PD Dr. K. Tenbrock}

Abteilung für Pädiatrische Rheumatologie, Klinik für Kinder und Jugendmedizin, Klinikum,

RWTH Aachen

Pauwelsstraße 30, 52074 Aachen

ktenbrock@ukaachen.de
Tab. 1 Zusammenfassung der Mechanismen, Indikationen und Dosierungen der vorgestellten Biologika

\begin{tabular}{|c|c|c|c|c|}
\hline Medikament & Mechanismus & Indikation & Dosierung & $\begin{array}{l}\text { Zulassung/ } \\
\text { EMA }\end{array}$ \\
\hline Etanercept & $\begin{array}{l}\text { Löslicher TNF- } \\
\text { Rezeptor }\end{array}$ & $\begin{array}{l}\text { Polyartikuläre JIA } \\
\text { Enthesitisassoziierte } \\
\text { JIA } \\
\text { Oligoartikuläre JIA }\end{array}$ & $\begin{array}{l}0,4 \text { mg/kg 2-mal wö- } \\
\text { chentlich s.c. }\end{array}$ & $\begin{array}{l}\text { Ja, für poly- } \\
\text { artikuläre JIA } \\
\text { ab dem 4. LJ }\end{array}$ \\
\hline Adalimumab & $\begin{array}{l}\text { Humanisierter } \\
\text { Anti-TNF-Anti- } \\
\text { körper }\end{array}$ & $\begin{array}{l}\text { Polyartikuläre JIA } \\
\text { Enthesitisassoziierte } \\
\text { JIA } \\
\text { Oligoartikuläre JIA } \\
\text { Uveitis }\end{array}$ & $\begin{array}{l}\text { Bei Patienten von } 4 \\
\text { bis } 12 \text { Jahren } 24 \mathrm{mg} / \\
\mathrm{m}^{2} \mathrm{KOF} \text {, maximal } 40 \mathrm{mg} \\
\text { Bei Patienten von } 12 \\
\text { bis } 17 \text { Jahren } 40 \mathrm{mg} \\
\text { alle } 2 \text { Wochen s.c. }\end{array}$ & $\begin{array}{l}\text { Ja, für poly- } \\
\text { artikuläre JIA } \\
\text { ab dem 4. LJ }\end{array}$ \\
\hline Infliximab & $\begin{array}{l}\text { Chimärer Maus- } \\
\text { Mensch-Anti- } \\
\text { körper }\end{array}$ & $\begin{array}{l}\text { Polyartikuläre JIA } \\
\text { Enthesitisassoziierte } \\
\text { JIA } \\
\text { Uveitis }\end{array}$ & $\begin{array}{l}6 \mathrm{mg} / \mathrm{kg} \text { in Wochen } \\
0,2,4,6 \text {, danach alle } \\
8 \text { Wochen }\end{array}$ & Nein \\
\hline Abatacept & $\begin{array}{l}\text { CTLA-4-Blockade } \\
\text { aufT-Zellen }\end{array}$ & Polyartikuläre JIA & $\begin{array}{l}10 \mathrm{mg} / \mathrm{kg} \text { in Wochen } 0, \\
2,4, \text { anschließend alle } \\
4 \text { Wochen }\end{array}$ & Ja, ab 6. LJ \\
\hline Anakinra & $\begin{array}{l}\text { IL-1-Rezeptoran- } \\
\text { tagonist }\end{array}$ & SOJIA & $1-2 \mathrm{mg} / \mathrm{kg}$ täglich s.c. & Nein \\
\hline Rilonacept & $\begin{array}{l}\text { Löslicher IL-1 } \beta \text { - } \\
\text { Rezeptor }\end{array}$ & SOJIA & 2,2 mg/Woche s.c. & Nein \\
\hline Canakinumab & $\begin{array}{l}\text { Humanisierter An- } \\
\text { ti-IL1-Antikörper }\end{array}$ & SOJIA & $\begin{array}{l}4 \mathrm{mg} / \mathrm{kg} 1 \text {-mal pro Mo- } \\
\text { nat s.c. }\end{array}$ & Nein \\
\hline Tocilizumab & $\begin{array}{l}\text { Anti-IL-6-Rezep- } \\
\text { tor-Antikörper }\end{array}$ & SOJIA & $\begin{array}{l}<20 \mathrm{~kg} 12 \mathrm{mg} / \mathrm{kg}, \\
>20 \mathrm{~kg} 8 \mathrm{mg} / \mathrm{kg} \text { alle } \\
2 \text { Wochen i.v. }\end{array}$ & $\mathrm{Ja}$ \\
\hline Rituximab & $\begin{array}{l}\text { Anti-CD20-Anti- } \\
\text { körper }\end{array}$ & $\begin{array}{l}\text { Polyarthritis und } \\
\text { SOJIA, die nicht auf } \\
\text { Anti-TNF-Therapie } \\
\text { angesprochen } \\
\text { haben }\end{array}$ & $\begin{array}{l}2 \text { Dosen a } 750 \mathrm{mg} / \mathrm{m}^{2} \\
\text { mit einem Abstand von } \\
2 \text { Wochen }\end{array}$ & Nein \\
\hline
\end{tabular}

CTLA "cytotoxic T-lymphocyte antigen“, EMA European Medicines Agency, IL Interleukin, JIA juvenile idiopathische Arthritis, KOF Körperoberfläche, LJ Lebensjahr, SOJIA systemische Form der JIA, TNF Tumornekrosefaktor 